Dept. of Math. University of Oslo

Pure Mathematics

No. 16

ISSN 0806-2439 May 2003

\title{
Malliavin Calculus and Anticipative Itô Formulae for Lévy Processes
}

\author{
Giulia Di Nunno ${ }^{1}$, Thilo Meyer-Brandis ${ }^{1}$, Bernt $\varnothing_{k s e n d a l}{ }^{1,2}$ and Frank Proske ${ }^{1}$
}

Oslo, 8th October 2004.

\begin{abstract}
We introduce the forward integral with respect to a pure jump Lévy process and we prove an Itô formula for this integral. Then we use Mallivin calculus to establish a relationship between the forward integral and the Skorohod integral and we apply this to obtain an Itô formula for the Skorohod integral.

Key words and phrases: Lévy processes, Poisson random measures, white noise, Skorohod integrals, forward integrals, stochastic derivatives, Wick product, anticipative Itô formulae.

AMS (2000) Classification: primary 60H40; secondary 60G51, 60G57, 60H07.

\section{Introduction.}

The original infinite dimensional calculus developed by Malliavin $[M]$ was designed to study the smoothness of the densities of the solutions of stochastic differential equations. Although this technique was developed further by many researchers, this application remained the only one known for several years. This situation changed in 1991, when Karatzas and Ocone [KO] showed how the representation theorem that Ocone had formulated some years earlier in terms of the Malliavin derivative could be used in finance. Now this theorem is often known as the Clark-Haussmann-Ocone (CHO) formula. More precisely, the CHO theorem gives a method of finding replicating portfolios in complete markets driven by Brownian motion. This discovery led to an enormous increase in the interest in the Malliavin calculus both among mathematicians and finance researchers and since then the theory has been generalized and new applications have been found. In particular, Malliavin calculus for Brownian motion has been applied to compute the greeks in finance, see e.g. [AØPU], [FLLLT] and [FLLL]. Moreover, anticipative stochastic caluclus for Brownian motion involving the forward integral (beyond the semimartingale context) has been applied to give a general approach to optimal portfolio and consumption problems for insiders in finance, see e.g. [BØ1], [BØ2] and [KS].

An extension of the Malliavin method to processes with discontinuous trajectories was carried out in 1987 by Bichteler, Gravereaux and Jacod [BGJ]. However, their work is focused on the original problem of the smoothness of the densities of the solutions of stochastic differential equations, a question that does not deal with the other more recent aspects of the Malliavin calculus. For related works on stochastic calculus for stochastic measures

\footnotetext{
${ }^{1}$ Centre of Mathematics for Applications (CMA), Department of Mathematics, University of Oslo, P.O. Box 1053 Blindern, N-0316 Oslo, Norway.

${ }^{2}$ Norwegian School of Economics and Business Administration, Helleveien 30, N-5045 Bergen, Norway.

E-mail addresses: giulian@math.uio.no; meyerbr@math.uio.no; oksendal@math.uio.no; proske@math.uio.no
} 
generated by a Poisson process on the real line see [DKW], [K1], [K2], [NV1], [Pi1] and [Pi2], for example.

Recently two types of Malliavin derivative operators $D_{t}^{(m)}$ and $D_{t, z}$ have been introduced for Lévy processes and the corresponding CHO representation theorems have been obtained. See $[\mathrm{L}],[\mathrm{LSUV}],[\mathrm{BDL} \varnothing \mathrm{P}],[\mathrm{D} \varnothing \mathrm{P}]$, and $[\varnothing \mathrm{P}]$. Although markets driven by Lévy processes are not in general complete, the corresponding $\mathrm{CHO}$ theorem is still important for financial applications. For example, it can be used to find explicitly the minimal variance portfolio (see e.g. [BDLØP]) and to compute the greeks in certain jump diffucsion market models (see e.g. [DJ]).

There has also been an increased interest in anticipative integration with respect to a Lévy process and this is partly due to its application to insider trading in finance (see e.g. $[\mathrm{DM} \varnothing \mathrm{P}],[\varnothing 2]$ and $[\varnothing \mathrm{S}])$. In Section 4 of this paper we introduce the forward integral with respect to a pure jump Lévy process and we prove an Itô formula for such integrals (see Theorem 4.7). Then we use a relation between forward integrals and Skorohod integrals (see Lemma 4.4) to obtain an Itô formula for Skorohod integrals with respect to a pure jump Lévy process (see Theorem 4.9).

Since Malliavin calculus plays a crucial role in our achievements, we give a review of the main results of this theory in Section 3. Various versions of those results have already been obtained and are known to the public. Nevertheless we think that it is of intrest to have a unified approach based on white noise theory.

For completeness and convenience of the reader we recall the basic theory of white noise for pure jump Lévy processes in Section 2.

\section{Framework.}

In this paper we deal with pure jump Lévy processes with no drift defined on a certain probability space $(\Omega, \mathcal{F}, P)$ and the time horizon $\mathbb{R}_{+}=[0, \infty)$. General information about Lévy processes can be found in $[\mathrm{Be}],[\mathrm{P}]$ and $[\mathrm{Sa}]$, for example. However we recall briefly our framework. Cf. [DØP], [ØР].

Let $\Omega=\mathcal{S}^{\prime}(\mathbb{R})$ be the Schwartz space of tempered distributions equipped with its Borel $\sigma$ algebra $\mathcal{F}=\mathfrak{B}(\Omega)$. The space $\mathcal{S}^{\prime}(\mathbb{R})$ is the dual of the Schwartz space $\mathcal{S}(\mathbb{R})$ of test functions, i.e. the rapidly decreasing smooth functions on $\mathbb{R}$. We denote the action of $\omega \in \Omega=\mathcal{S}^{\prime}(\mathbb{R})$ applied to $f \in \mathcal{S}(\mathbb{R})$ by $\langle\omega, f\rangle=\omega(f)$. See $[G V]$, for example.

Thanks to the Bochner-Milnos-Sazonov theorem, the white noise probability measure $P$ can be defined by the relation

$$
\int_{\Omega} e^{i\langle\omega, f\rangle} d P(\omega)=e^{\int_{\mathbb{R}} \psi(f(x)) d x-i \alpha \int_{\mathbb{R}} f(x) d x}, \quad f \in \mathcal{S}(\mathbb{R}),
$$

where the real constant $\alpha$ and

$$
\psi(u)=\int_{\mathbb{R}}\left(e^{i u z}-1-i u z 1_{\{|z|<1\}}\right) \nu(d z)
$$


are the elements of the exponent in the characteristic functional of a pure jump Lévy process with the Lévy measure $\nu(d z), z \in \mathbb{R}$, which, we recall, is such that

$$
\int_{\mathbb{R}} 1 \wedge z^{2} \nu(d z)<\infty
$$

Assuming that

$$
M:=\int_{\mathbb{R}} z^{2} \nu(d z)<\infty,
$$

we can set $\alpha=\int_{\mathbb{R}} z 1_{\{|z|>1\}} \nu(d z)$ and then we obtain that

$$
E[\langle\cdot, f\rangle]=0 \quad \text { and } \quad E\left[\langle\cdot, f\rangle^{2}\right]=M \int_{\mathbb{R}} f(x) d x, \quad f \in \mathcal{S}(\mathbb{R}) .
$$

Accordingly the pure jump Lévy process with no drift

$$
\eta=\eta(\omega, t), \quad \omega \in \Omega, t \in \mathbb{R}_{+},
$$

that we do consider here and in the sequel, is the cadlag modification of $\left\langle\omega, \chi_{(0, t]}\right\rangle, \omega \in \Omega$, $t>0$, where

$$
\chi_{(0, t]}(x)=\left\{\begin{array}{lc}
1, & 0<x \leq t \\
0, & \text { otherwise, } \quad x \in \mathbb{R},
\end{array}\right.
$$

with $\eta(\omega, 0):=0, \omega \in \Omega$. We remark that, for all $t \in \mathbb{R}_{+}$, the values $\eta(t)$ belong to $L^{2}(P):=L^{2}(\Omega, \mathcal{F}, P)$.

The Lévy process $\eta$ can be expressed by

$$
\eta(t)=\int_{0}^{\infty} \int_{\mathbb{R}} z \widetilde{N}(d t, d z), \quad t \in \mathbb{R}_{+},
$$

where $\widetilde{N}(d t, d z):=N(d t, d z)-\nu(d z) d t$ is the compensated Poisson random measure associated with $\eta$ - cf. [I1], for example. Recall that the Poisson process is the most important representative among the pure jump Lévy processes and it corresponds to the specific case in which the measure $\nu$ is a point mass at 1 .

Let $\mathcal{F}_{t}, t \in \mathbb{R}_{+}$, be the completed filtration generated by the Lévy process in (2.4). We fix $\mathcal{F}=\mathcal{F}_{\infty}$.

Aiming to treat the Malliavin calculus by means of chaos expansions, we now recall the required spaces and the corresponding complete orthonormal systems.

In the space $L^{2}(\lambda)=L^{2}\left(\mathbb{R}_{+}, \mathfrak{B}\left(\mathbb{R}_{+}\right), \lambda\right)$ of the square integrable functions on $\mathbb{R}_{+}$equipped with the Borel $\sigma$-algebra and the standard Lebesgue measure $\lambda(d t), t \in \mathbb{R}_{+}$, we consider the complete orthonormal system $\xi_{j}(j=1,2, \ldots)$ of the Laguerre functions of order $1 / 2$, i.e.

$$
\xi_{j}(t)=\left(\frac{\Gamma(j)}{\Gamma(j+1 / 2)}\right)^{1 / 2} e^{-t} t^{1 / 4} L_{j-1}^{1 / 2}(t) 1_{(0, \infty)}(t), \quad t \in \mathbb{R}_{+} \quad(j=1,2, \ldots),
$$

where $\Gamma$ is the Gamma functions and $L_{j}^{1 / 2}$ are the Laguerre polynomials of order $1 / 2$ defined by

$$
e^{-t} t^{1 / 2} L_{j}^{1 / 2}(t)=\frac{1}{j !} \frac{d^{j}}{d t^{j}}\left(e^{-t} t^{j+1 / 2}\right) \quad(j=0,1, \ldots) .
$$


Cf. [T], for example.

In the space $L^{2}(\nu):=L^{2}(\mathbb{R}, \mathfrak{B}(\mathbb{R}), \nu)$ of the square integrable functions on $\mathbb{R}$ equipped with the Borel $\sigma$-algebra and the Lévy measure $\nu$, we fix a complete orthonormal system $\psi_{i}(i=1,2, \ldots)$. In particular we can choose a complete system of polynomials as it was suggested in $[\mathrm{NS}]$ and $[\mathrm{D} \emptyset \mathrm{P}]$, provided that the moments of order grater than or equal to 2 of the measure $\nu$ are finite.

To simplify the notation we call $\mathcal{J}$ the set of multi-indexes $\alpha=\left(\alpha_{1}, \alpha_{2}, \ldots\right)$ which have only finitely many non-zero values. We denote $\operatorname{Index}(\alpha)=\max \left\{n: \alpha_{n} \neq 0\right\}$ and $|\alpha|=\sum_{n} \alpha_{n}$, for $\alpha \in \mathcal{J}$.

By $\delta_{k}(k=1,2, \ldots)$ we identify the product

$$
\delta_{k}(t, z):=\xi_{j}(t) \psi_{i}(z), \quad t \in \mathbb{R}_{+}, z \in \mathbb{R},
$$

where $k=\gamma(i, j)$, and $\gamma: \mathbb{N} \times \mathbb{N} \longrightarrow \mathbb{N}$ is a bijective map. Note that any bijective map can be applied, e.g. we could consider the so-called "Cantor diagonalization" of the Cartesian product $\mathbb{N} \times \mathbb{N}$. We set

$$
\epsilon^{k}(n)=\left\{\begin{array}{ll}
1, & n=k \\
0, & \text { otherwise }
\end{array} \quad(k=1,2, \ldots) .\right.
$$

Now, for any $\alpha \in \mathcal{J}$ with $\operatorname{Index}(\alpha)=n$ and $|\alpha|=m$ we define the tensor product $\delta^{\otimes \alpha}$ as

$$
\begin{gathered}
\delta^{\otimes \alpha}=\delta_{1}^{\otimes \alpha_{1}} \otimes \ldots \otimes \delta_{n}^{\otimes \alpha_{n}}\left(\left(t_{1}, x_{1}\right), \ldots,\left(t_{m}, x_{m}\right)\right) \\
:=\delta_{1}\left(t_{1}, x_{1}\right) \cdot \ldots \cdot \delta_{1}\left(t_{\alpha_{1}}, x_{\alpha_{1}}\right) \cdot \ldots \cdot \delta_{n}\left(t_{\alpha_{1}+\ldots+\alpha_{n-1}+1}, x_{\alpha_{1}+\ldots+\alpha_{n-1}+1}\right) \cdot \ldots \cdot \delta_{n}\left(t_{m}, x_{m}\right),
\end{gathered}
$$

with $\delta_{k}^{\otimes 0}:=1$. Then we denote $\delta^{\widehat{\otimes} \alpha}$ the symmetrization of the functions $\delta_{k}^{\otimes \alpha}$.

In the space $L^{2}(P):=L^{2}(\Omega, \mathcal{F}, P)$ of the square integrable random variables we consider the following complete orthogonal system $K_{\alpha}(\alpha \in \mathcal{J})$ :

$$
K_{\alpha}:=I_{|\alpha|}\left(\delta^{\widehat{\otimes} \alpha}\right), \quad \alpha \in \mathcal{J},
$$

where

$I_{m}(f):=m ! \int_{0}^{\infty} \int_{\mathbb{R}} \ldots \int_{0}^{t_{2}} \int_{\mathbb{R}} f\left(t_{1}, x_{1}, \ldots, t_{m}, x_{m}\right) \tilde{N}\left(d t_{1}, d x_{1}\right) \ldots \tilde{N}\left(d t_{m}, d x_{m}\right) \quad(m=1,2, \ldots)$

for the symmetric function $f \in L^{2}\left((\lambda \times \nu)^{m}\right)(m=1,2, \ldots)$ and $I_{0}(f):=f$ for $f \in \mathbb{R}$. Cf. $[\mathrm{D} \varnothing \mathrm{P}]$ and $[\varnothing \mathrm{P}]$. In particular $K_{\epsilon^{k}}=I_{1}\left(\delta_{k}\right)$. Note that $\left\|K_{\alpha}\right\|_{L^{2}(P)}^{2}=\alpha !=\alpha_{1} ! \alpha_{2} ! \ldots(\alpha \in \mathcal{J})$.

The following result is given in $[\mathrm{D} \varnothing \mathrm{P}]$, see also $[\varnothing \mathrm{P}]$.

Theorem 2.1 (Chaos expansion I). Every $F \in L^{2}(P)$ admits the unique representation in the form

$$
F=\sum_{\alpha \in \mathcal{J}} c_{\alpha} K_{\alpha}
$$

where $c_{\alpha} \in \mathbb{R}$ for all $\alpha \in \mathcal{J}$, and $c_{0}=E F$. Moreover we have

$$
\|F\|_{L^{2}(P)}^{2}=\sum_{\alpha \in \mathcal{J}} c_{\alpha} \alpha !
$$


If we consider the symmetric functions

$$
f_{m}=\sum_{\alpha:|\alpha|=m} c_{\alpha} \delta^{\widehat{\otimes} \alpha} \quad(m=1,2, \ldots)
$$

then we obtain

$$
\sum_{\alpha \in \mathcal{J}} c_{\alpha} K_{\alpha}=\sum_{m=0}^{\infty} \sum_{\alpha:|\alpha|=m} c_{\alpha} I_{m}\left(\delta^{\widehat{\otimes} \alpha}\right)=\sum_{m=0}^{\infty} I_{m}\left(f_{m}\right) .
$$

The expansion here above is actually a result which was first proved in [I2]. We can state it as follows.

Theorem 2.2 (Chaos expansion II). Every $F \in L^{2}(P)$ admits the (unique) representation

$$
F=\sum_{m=0}^{\infty} I_{m}\left(f_{m}\right)
$$

via the unique sequence of symmetric functions $f_{m} \in L^{2}\left((\lambda \times \nu)^{m}\right), m=0,1, \ldots$.

For any formal expansion $f=\sum_{\alpha \in \mathcal{J}} c_{\alpha} K_{\alpha}$ we define the norm

$$
\|f\|_{0, k}^{2}:=\sum_{\alpha \in \mathcal{J}} \alpha ! c_{\alpha}^{2}(2 \mathbb{N})^{k \alpha} \quad(k=0,1, \ldots)
$$

where $(2 \mathbb{N})^{k \alpha}=(2 \cdot 1)^{k \alpha_{1}}(2 \cdot 2)^{k \alpha_{2}}(2 \cdot 3)^{k \alpha_{3}} \ldots$ Now setting

$$
(\mathcal{S})_{0, k}:=\left\{f:\|f\|_{0, k}<\infty\right\}
$$

we define

$$
(\mathcal{S}):=\bigcap_{k=0}^{\infty}(\mathcal{S})_{0, k}
$$

with the projective topology, and

$$
(\mathcal{S})^{*}:=\bigcup_{k=0}^{\infty}(\mathcal{S})_{0, k}
$$

with the inductive topology. The space $(\mathcal{S})^{*}$ is the dual of $(\mathcal{S})$ and the action of $G=$ $\sum_{\alpha \in \mathcal{J}} a_{\alpha} K_{\alpha} \in(\mathcal{S})^{*}$ applied to $f=\sum_{\alpha \in \mathcal{J}} b_{\alpha} K_{\alpha} \in(\mathcal{S})$ is

$$
\langle G, f\rangle=\sum_{\alpha \in \mathcal{J}} a_{\alpha} b_{\alpha} \alpha !
$$

Note that

$$
(\mathcal{S}) \subset L^{2}(P) \subset(\mathcal{S})^{*} .
$$

We refer to [HKPS] and [HoØUZ], for example, for the above definitions in the setting of the Gaussian and Poissonian white noise. See also $[\mathrm{PT}],[\mathrm{BG}],[\mathrm{Ho} \emptyset]$ and references therein. For the Lévy case, we refer to [DØP]. 
Definition 2.3 The white noise $\dot{\widetilde{N}}(t, x)$ of the Poisson random measure $\tilde{N}(d t, d z)$ is defined by the following formal expansion

$$
\dot{\widetilde{N}}(t, z)=\sum_{i, j=1}^{\infty} \xi_{i}(t) \psi_{j}(z) \cdot K_{\epsilon^{\gamma(i, j)}} .
$$

It can be proved that the white noise takes values in $(\mathcal{S})^{*},(\lambda \times \nu)$-a.e. See $[\varnothing \mathrm{P}]$. The justification of the name "white noise" comes from the fact that, for any $B \in \mathfrak{B}(\mathbb{R})$ such that its closure does not contain 0 , we have

$$
I_{1}\left(\chi_{(0, t]} 1_{B}\right)=\sum_{i, j=1}^{\infty} c_{\gamma(i, j)} K_{\epsilon^{\gamma(i, j)}}
$$

with $c_{\gamma(i, j)}=\int_{0}^{t} \int_{B} \xi_{i}(s) \psi_{j}(z) \nu(d z) d s \cdot K_{\epsilon \gamma(i, j)}$. Cf. (2.8) and (2.10). Then

$$
\tilde{N}(t, B)=\int_{0}^{t} \int_{B}\left(\sum_{i, j=1}^{\infty} \xi_{i}(s) \psi_{j}(z) K_{\epsilon^{\gamma(i, j)}}\right) \nu(d z) d s .
$$

So formally we have

$$
\stackrel{\tilde{\widetilde{N}}}{(t, z)}=\frac{\widetilde{N}(d t, d z)}{d t \nu(d z)}
$$

which is the analog of the Radon-Nikodym derivative in $(\mathcal{S})^{*}$.

Definition 2.4 The white noise $\dot{\eta}(t)$ for the Lévy process is defined by the following formal expansion

$$
\dot{\eta}(t)=\sum_{i=1}^{\infty} \xi_{i}(t) K_{\epsilon^{\gamma(i, 1)}},
$$

for a specific choice of the basis $\psi_{i}, i=1,2, \ldots$, in $(2.6)$ - cf. [DØP].

The Lévy white noise takes values in $(\mathcal{S})^{*}$ for all $t \in \mathbb{R}_{+}$. Here the boundedness of the Laguerre functions can be exploited, cf. [T].

Note that the Lévy white noise (2.13) is related to the white noise for the Poisson random measure (2.12) by the following formula which involves Bochner integrals with respect to $\nu$ :

$$
\dot{\eta}(t)=\int_{\mathbb{R}} z \stackrel{\mathscr{N}}{N}(t, z) \nu(d z) .
$$

Definition 2.5 The Wick product $F \diamond G$ of two elements $F=\sum_{\alpha \in \mathcal{J}} a_{\alpha} K_{\alpha}$ and $G=$ $\sum_{\beta \in \mathcal{J}} b_{\beta} K_{\beta}$ in $(\mathcal{S})^{*}$ is defined by

$$
F \diamond G=\sum_{\alpha, \beta \in \mathcal{J}} a_{\alpha} b_{\beta} K_{\alpha+\beta}
$$

The spaces $(\mathcal{S})$ and $(\mathcal{S})^{*}$ are topological algebras with respect to the Wick product. 


\section{Some anticipative calculus formulae.}

In this section we present some known formulae for the Malliavin calculus in the case of pure jump Lévy processes. We will need these results in Section 4. These formulae generalize the known results for the Malliavin calculus in the case of Brownian motion, cf. $[\mathrm{NP}],[\mathrm{M}],[\mathrm{N}]$, $[\varnothing]$, for example. First we recall the Skorohod integration and the Malliavin type stochastic derivative we are dealing with.

Let $X(t, z), t \in \mathbb{R}_{+}, z \in \mathbb{R}$, be a random field taking values in $L^{2}(P)$. Then, for all $t \in \mathbb{R}_{+}$ and $z \in \mathbb{R}$, Theorem 2.2 provides the chaos expansion via symmetric functions

$$
X(t, z)=\sum_{m=0}^{\infty} I_{m}\left(f_{m}\left(t_{1}, z_{1}, \ldots, t_{m}, z_{m} ; t, z\right)\right)
$$

Let $\widehat{f}_{m}=\widehat{f}_{m}\left(t_{1}, z_{1}, \ldots, t_{m+1}, z_{m+1}\right)$ be the symmetrization of $f_{m}\left(t_{1}, z_{1}, \ldots, t_{m}, z_{m} ; t, z\right)$ as a function of the $m+1$ variables $\left(t_{1}, z_{1}\right), \ldots,\left(t_{m+1}, z_{m+1}\right)$ with $t_{m+1}=t$ and $z_{m+1}=z$.

The following concept was first introduced by Y. Kabanov - see [Ka1] and [Ka2], for example.

Definition 3.1 The random field $X(t, z), t \in \mathbb{R}_{+}, z \in \mathbb{R}$, is Skorohod integrable if $\sum_{m=0}^{\infty}(m+1) !\left\|\widehat{f}_{m}\right\|_{L^{2}\left((\lambda \times \nu)^{m+1}\right)}^{2}<\infty$. Then its Skorohod integral with respect to $\widetilde{N}$, i.e.

$$
I(X):=\int_{\mathbb{R}_{+}} \int_{\mathbb{R}} X(t, z) \tilde{N}(\delta t, d z),
$$

is defined by

$$
I(X):=\sum_{m=0}^{\infty} I_{m+1}\left(\widehat{f}_{m}\right) .
$$

The Skorohod integral is an element of $L^{2}(P)$ and

$$
\left\|\int_{\mathbb{R}_{+}} \int_{\mathbb{R}} X(t, z) \tilde{N}(\delta t, d z)\right\|_{L^{2}(P)}^{2}=\sum_{m=0}^{\infty}(m+1) !\left\|\widehat{f}_{m}\right\|_{L^{2}\left((\lambda \times \nu)^{m+1}\right)}^{2} .
$$

Moreover,

$$
E \int_{\mathbb{R}_{+}} \int_{\mathbb{R}} X(t, z) \widetilde{N}(\delta t, d z)=0 .
$$

The Skorohod integral can be regarded as an extension of the Itô integral to anticipative integrands. In fact, the following result can be proved. Cf. [NV2]. See also [BL], [DØP] and $[\varnothing \mathrm{P}]$.

Proposition 3.2 Let $X(t, z), t \in \mathbb{R}_{+}, z \in \mathbb{R}$, be a non-anticipative (adapted) integrand. Then the Skorohod integral and the Itô integral coincide in $L^{2}(P)$, i.e.

$$
\int_{\mathbb{R}_{+}} \int_{\mathbb{R}} X(t, z) \tilde{N}(\delta t, d z)=\int_{\mathbb{R}_{+}} \int_{\mathbb{R}} X(t, z) \tilde{N}(d t, d z) .
$$


Another remarkable property of the Skorohod integral is given in connection to the Wick product. See $[\varnothing \mathrm{P}]$ for the details and the proof. Before stating the result, we remind that a random field $Y$ taking values in $(\mathcal{S})^{*}$ is said to be $(\mathcal{S})^{*}$-integrable whenever $\langle Y, f\rangle \in L^{1}(\lambda \times \nu)$ for all $f \in(\mathcal{S})$.

Definition 3.3 For any $(\mathcal{S})^{*}$-integrable random field $Y(t, z)$ the $(\mathcal{S})^{*}$-integral is the unique element in $(\mathcal{S})^{*}$ such that

$$
\left\langle\int_{\mathbb{R}_{+}} \int_{\mathbb{R}} Y(t, z) \nu(d z) d t, f\right\rangle=\int_{\mathbb{R}_{+}} \int_{\mathbb{R}}\langle Y(t, z), f\rangle \nu(d z) d t, \quad f \in(\mathcal{S}) .
$$

Theorem 3.4 Let $Y(t, z), t \in \mathbb{R}_{+}, z \in \mathbb{R}$, be Skorohod integrable and $\int_{a}^{b} \int_{\mathbb{R}} E\left[Y(t, z)^{2}\right] \nu(d z) d t$ $<\infty$ for some $0 \leq a<b$. Then $Y \diamond \dot{\widetilde{N}}$ is $(\mathcal{S})^{*}-$ integrable over $[a, b] \times \mathbb{R}$ and we obtain the following relationship

$$
\int_{a}^{b} \int_{\mathbb{R}} Y(t, z) \widetilde{N}(\delta t, d z)=\int_{a}^{b} \int_{\mathbb{R}} Y(t, z) \diamond \dot{\widetilde{N}}(t, z) \nu(d z) d t .
$$

Thanks to the relation (2.4), we can easily recognize the Skorohod integral with respect to the very Lévy process $\eta(t), t \in \mathbb{R}_{+}$, as a particular case of the Skorohod integration with respect to the compensated Poisson random measure $\widetilde{N}$. See $[\mathrm{D} \varnothing \mathrm{P}]$. In fact, for the integrands $X(t, z)=z \cdot \varphi(t): X(t, z)=\sum_{m=0}^{\infty} I_{m}\left(z \cdot f\left(t_{1}, z_{1}, \ldots, t_{m}, z_{m} ; t\right)\right)$, we have

$$
\int_{0}^{\infty} \int_{\mathbb{R}} X(t, z) \tilde{N}(\delta t, d z)=\int_{\mathbb{R}} \varphi(t) \delta \eta(t) .
$$

Now we consider the definition of the Malliavin type derivative $D_{t, z}$ for compensated Poisson random measures which was initially given in [L]. Other definitions have also been studied by several authors for the same case of pure jump Lévy processes, the particular case of Poisson random processes and for the case of the general Lévy process with no drift, see for instance [BC], [Pi1], [Pi2], [NV1], [NV2], [NS], [LSUV], [DØP] and [BDLØP].

Definition 3.5 The space $D_{1,2}$ is the set of all the elements $F \in L^{2}(P)$ admitting the chaos expansion (2.11): $F=E[F]+\sum_{m=1}^{\infty} I_{m}\left(f_{m}\right)$, such that

$$
\|F\|_{\mathbb{D}_{1,2}}^{2}:=\sum_{m=1}^{\infty} m \cdot m !\left\|f_{m}\right\|_{L^{2}\left((\lambda \times \nu)^{m}\right)}^{2}<\infty .
$$

The Malliavin derivative $D_{t, z}$ is an operator defined on $D_{1,2}$ with values in the standard $L^{2}$-space $L^{2}(P \times \lambda \times \nu)$ given by

$$
D_{t, z} F:=\sum_{m=1}^{\infty} m I_{m-1}\left(f_{m}(\cdot, t, z)\right),
$$

where $f_{m}(\cdot, t, z)=f_{m}\left(t_{1}, z_{1}, \ldots, t_{m-1}, z_{m-1} ; t, z\right)$. 
Note that if $F \in \mathbb{D}_{1,2}$, then

$$
E\left[\int_{0}^{\infty} \int_{\mathbb{R}}\left(D_{t, z} F\right)^{2} \nu(d z) d t\right]=\|F\|_{\mathbb{D}_{1,2}}^{2} .
$$

The operator $D_{t, z}$ is proved to be closed and to coincide with a certain difference operator defined in [Pi1]. $(2.9)$.

The above operator can be extended to the whole space $(\mathcal{S})^{*}$ thanks to the chaos expansion

Definition 3.6 For any $F=\sum_{\alpha \in \mathcal{J}} c_{\alpha} K_{\alpha} \in(\mathcal{S})^{*}$ the Malliavin derivative $D_{t, z} F$ is defined as

$$
D_{t, z} F:=\sum_{\alpha \in \mathcal{J}} c_{\alpha} \sum_{i, j=1}^{\infty} \alpha_{\gamma(i, j)} K_{\alpha-\epsilon^{\gamma(i, j)}} \cdot \xi_{i}(t) \psi_{j}(z) .
$$

It can be proved that $D_{t, z} F \in(\mathcal{S})^{*}, \lambda \times \nu$-a.e., for all $F \in(\mathcal{S})^{*}$. Moreover it can also be shown that if $F=\lim _{n \rightarrow \infty} F_{n}$ in $(\mathcal{S})^{*}$, then there exists a subsequence $F_{n_{k}} \in(\mathcal{S})^{*}$ such that $D_{t, z} F=\lim _{n \rightarrow \infty} D_{t, z} F_{n_{k}}$ in $(\mathcal{S})^{*}, \lambda \times \nu$-a.e. See $[\varnothing \mathrm{P}]$.

We remark that in general the stochastic derivative $D_{t, z}$, being essentially a difference operator, does not satisfy a "chain rule" as in the case of the Malliavin derivative for the Brownian motion setting. Cf. $[\mathrm{NP}],[\mathrm{N}],[\varnothing]$, for example. Nevertheless a "chain rule" can still be formulated in terms of the Wick product.

Proposition 3.7 (Chain rule via Wick product). Let $F \in(\mathcal{S})^{*}$ and let $g(z)=\sum_{n \geq 0} a_{n} z^{n}$ be an analytic function in the whole complex plane. Then $\sum_{n \geq 0} a_{n} F^{\diamond n}$ is convergent in $(\mathcal{S})^{*}$. Furthermore, for $g^{\diamond}(F)=\sum_{n \geq 0} a_{n} F^{\diamond n}$, the following Wick chain rule is valid

$$
D_{s, x} g^{\diamond}(F)=\left(\frac{d}{d z} g\right)^{\diamond}(F) \diamond D_{s, x} F .
$$

Proof. The first statement can be derived following similar proofs as in Theorem 2.6.12 and

Theorem 2.8.1 in [HØUZ]. For what concerns the chain rule, it can be easily shown that it holds for polynomials. Then the result follows by the closeness of $D_{t, z}$ and the continuity of the Wick product.

Now we turn our attention more deeply to the calculus and we present some basic explicit formulae. First of all we recall the following result proved in [BL]. See also [DJ] Theorem 2.6 .

Theorem 3.8 (Duality formula). Let $X(t, z), t \in \mathbb{R}_{+}, z \in \mathbb{R}$, be Skorohod integrable and $F \in \mathbb{D}_{1,2}$. Then

$$
E\left[\int_{0}^{\infty} \int_{\mathbb{R}} X(t, z) D_{t, z} F \nu(d z) d t\right]=E\left[F \int_{0}^{\infty} \int_{\mathbb{R}} X(t, z) \widetilde{N}(\delta t, d z)\right] .
$$


Corollary 3.9 (Closability of Skorohod integral). Suppose that $X_{n}(t, z), t \in \mathbb{R}_{+}, z \in$ $\mathbb{R}$, is a sequence of Skorohod integrable random fields and that the corresponding sequence of integrals

$$
I\left(X_{n}\right):=\int_{0}^{\infty} \int_{\mathbb{R}} X_{n}(t, z) \widetilde{N}(\delta t, d z), \quad n=1,2, \ldots
$$

converges in $L^{2}(P)$. Moreover suppose that

$$
\lim _{n \rightarrow \infty} X_{n}=0 \quad \text { in } \quad L^{2}(P \times \lambda \times \nu) .
$$

Then we have

$$
\lim _{n \rightarrow \infty} I\left(X_{n}\right)=0 \quad \text { in } \quad L^{2}(P) .
$$

Proof. By Theorem (3.8) we have that

$$
\left(I\left(X_{n}\right), F\right)_{L^{2}(P)}=\left(X_{n}, D_{t, z} F\right)_{L^{2}(P \times \lambda \times \nu)} \longrightarrow 0, \quad n \rightarrow \infty
$$

for all $F \in \mathbb{D}_{1,2}$. Then we conclude that $\lim _{n \rightarrow \infty} I\left(X_{n}\right)=0$ weakly in $L^{2}(P)$. And since the sequence $I\left(X_{n}\right), n=1,2, \ldots$, is convergent in $L^{2}(P)$, then the result follows.

In view of Corollary 3.9 we can extend the definition of Skorohod integral as follows.

Definition 3.10 Let $X_{n}, n=1,2, \ldots$, be a sequence of Skorohod integrable random fields such that

$$
X=\lim _{n \rightarrow \infty} X_{n} \quad \text { in } \quad L^{2}(P \times \lambda \times \nu) .
$$

Then we define the Skorohod integral of $X$ as

$$
I(X):=\int_{0}^{\infty} \int_{\mathbb{R}} X(t, z) \tilde{N}(\delta t, d z)=\lim _{n \rightarrow \infty} \int_{0}^{\infty} \int_{\mathbb{R}} X_{n}(t, z) \tilde{N}(\delta t, d z)=: \lim _{n \rightarrow \infty} I\left(X_{n}\right),
$$

provided that this limit exists in $L^{2}(P)$.

The following result is Lemma 6.1 in [NV1] (there obtained in a more general setting).

Lemma 3.11 Let $F, G \in \mathbb{D}_{1,2}$ with $G$ bounded. Then $F \cdot G \in \mathbb{D}_{1,2}$ and we have

$$
D_{t, z}(F \cdot G)=F \cdot D_{t, z} G+G \cdot D_{t, z} F+D_{t, z} F \cdot D_{t, z} G \quad \lambda \times \nu-\text { a.e. }
$$

Proof. With the help of Lemma 9 in [L] the result can be verified for $F$ and $G$ of the form $g\left(\eta\left(t_{1}\right), \ldots, \eta\left(t_{k}\right)\right)$, where $g$ is a smooth function with compact support. Then, by using a limit argument the proof follows from the closedness of $D_{t, z}$.

Remark 3.12 For an extension of this result to normal martingales, see for example Proposition 1 in [Pr2] or Proposition 5 in [PSV].

The following result is Theorem 7.1 in [NV1] (there obtained in a more general setting). 
Theorem 3.13 (Integration by parts). Let $X(t, z), t \in \mathbb{R}_{+}, z \in \mathbb{R}$, be a Skorohod integrable stochastic process and $F \in \mathbb{D}_{1,2}$ such that the product $X(t, z) \cdot\left(F+D_{t, z} F\right)$, $t \in \mathbb{R}_{+}, z \in \mathbb{R}$, is Skorohod integrable. Then

$$
\begin{gathered}
F \int_{0}^{\infty} \int_{\mathbb{R}} X(t, z) \tilde{N}(\delta t, d z) \\
=\int_{0}^{\infty} \int_{\mathbb{R}} X(t, z)\left(F+D_{t, z} F\right) \tilde{N}(\delta t, d z)+\int_{0}^{\infty} \int_{\mathbb{R}} X(t, z) D_{t, z} F \nu(d z) d t .
\end{gathered}
$$

Proof. Let $G \in \mathbb{D}_{1,2}$ be bounded. Then we obtain by Theorem 3.8 and Lemma 3.11

$$
\begin{gathered}
E\left[G \int_{0}^{\infty} \int_{\mathbb{R}} F X(t, z) \tilde{N}(\delta t, d z)\right]=E\left[\int_{0}^{\infty} \int_{\mathbb{R}} F X(t, z) D_{t, z} G \nu(d z) d t\right] \\
=E\left[G F \int_{0}^{\infty} \int_{\mathbb{R}} X(t, z) \tilde{N}(\delta t, d z)\right]-E\left[G \int_{0}^{\infty} \int_{\mathbb{R}} X(t, z) D_{t, z} F \nu(d z) d t\right] \\
-E\left[G \int_{0}^{\infty} \int_{\mathbb{R}} X(t, z) D_{t, z} F \tilde{N}(\delta t, d z)\right] \\
=E\left[G \left(F \int_{0}^{\infty} \int_{\mathbb{R}} X(t, z) \tilde{N}(\delta t, d z)-\int_{0}^{\infty} \int_{\mathbb{R}} X(t, z) D_{t, z} F \nu(d z) d t\right.\right. \\
\left.\left.-\int_{0}^{\infty} \int_{\mathbb{R}} X(t, z) D_{t, z} F \tilde{N}(\delta t, d z)\right)\right] .
\end{gathered}
$$

The proof then follows by a density argument applied to $G$.

Remark 3.14 Using the Poisson interpretation of Fock space, the formula (3.12) has been shown to be an expression of the multiplication formula for Poisson stochastic integrals. See [Ka2], [Su], Proposition 2 and Relation (6) of [Pr3], Definition 7 and Proposition 6 of [PW], Proposition 2 of [PSV] and Proposition 1 of [Pr1].

Formula (3.12) has been known for some time to quantum probabilitsts in identical or close formulations. See Proposition 21.6 and Proposition 21.8 in [Pa], Proposition 18 in [B] and Relation (5.6) in $[\mathrm{A}]$.

The following result is Theorem 4.2 in [NV1] (there obtained in a more general setting).

Theorem 3.15 (Fundamental theorem of calculus). Let $X \in L^{2}(P \times \lambda \times \nu)$. Assume that $X(s, y) \in D_{1,2}$ for all $(s, y)$, and $D_{t, z} X(s, y), s \in \mathbb{R}_{+}, y \in \mathbb{R}$, for $(t, z) \lambda \times \nu$-a.e. is Skorohod integrable and that

$$
E\left[\int_{0}^{\infty} \int_{\mathbb{R}}\left(\int_{0}^{\infty} \int_{\mathbb{R}} D_{t, z} X(s, y) \tilde{N}(\delta s, d y)\right)^{2} \nu(d z) d t\right]<\infty .
$$

Then $\int_{0}^{\infty} \int_{\mathbb{R}} X(s, y) \widetilde{N}(\delta s, d y) \in \mathbb{D}_{1,2}$ and

$$
D_{t, z}\left(\int_{0}^{\infty} \int_{\mathbb{R}} X(s, y) \tilde{N}(\delta s, d y)\right)=\int_{0}^{\infty} \int_{\mathbb{R}} D_{t, z} X(s, y) \tilde{N}(\delta s, d y)+X(t, z) .
$$


Proof. First suppose that

$$
X(s, y)=I_{n}\left(f_{n}(\cdot, s, y)\right),
$$

where $f_{n}\left(t_{1}, z_{1}, \ldots, t_{n}, z_{n}, s, y\right)$ is symmetric with respect to $\left(t_{1}, z_{1}\right), \ldots,\left(t_{n}, z_{n}\right)$. By Definition 3.1 we have

$$
\int_{0}^{\infty} \int_{\mathbb{R}} X(s, y) \tilde{N}(\delta s, d y)=I_{n+1}\left(\widehat{f_{n}}\right)
$$

where

$$
\begin{gathered}
\widehat{f_{n}}\left(t_{1}, z_{1}, \ldots, t_{n}, z_{n}, t_{n+1}, z_{n+1}\right) \\
=\frac{1}{n+1}\left[f_{n}\left(t_{n+1}, z_{n+1}, \cdot, t_{1}, z_{1}\right)+\ldots+f_{n}\left(t_{n+1}, z_{n+1}, \cdot, t_{n}, z_{n}\right)+f_{n}\left(t_{1}, z_{1}, \cdot, t_{n+1}, z_{n+1}\right)\right]
\end{gathered}
$$

is the symmetrization of $f_{n}$ with respect to the variables $\left(t_{1}, z_{1}\right), \ldots,\left(t_{n}, z_{n}\right),\left(t_{n+1}, z_{n+1}\right)=$ $(s, y)$. Therefore we get

$$
D_{t, z}\left(\int_{0}^{\infty} \int_{\mathbb{R}} X(s, y) \tilde{N}(\delta s, d y)\right)=I_{n}\left(f_{n}\left(t, z, \cdot, t_{1}, z_{1}\right)+\ldots+f_{n}\left(t, z, \cdot, t_{n}, z_{n}\right)+f_{n}(\cdot, t, z)\right) .
$$

On the other hand we see that

$$
\begin{gathered}
\int_{0}^{\infty} \int_{\mathbb{R}} D_{t, z} X(s, y) \widetilde{N}(\delta s, d y) \\
=\int_{0}^{\infty} \int_{\mathbb{R}} n I_{n-1}\left(f_{n}(\cdot, t, z, s, y)\right) \tilde{N}(\delta s, d y)=n I_{n}\left(\widehat{f_{n}}(\cdot, t, z, \cdot)\right),
\end{gathered}
$$

where

$$
\widehat{f_{n}}\left(t_{1}, z_{1}, \ldots, t_{n-1}, t_{n-1}, t, z, t_{n}, z_{n}\right)=\frac{1}{n}\left[f_{n}\left(t, z, \cdot, t_{1}, z_{1}\right)+\ldots+f_{n}\left(t, z, \cdot, t_{n}, z_{n}\right)\right]
$$

is the symmetrization of $f_{n}\left(t_{1}, z_{n}, \ldots, t_{n-1}, z_{n-1}, t, z, t_{n}, z_{n}\right)$ with respect to $\left(t_{1}, z_{1}\right), \ldots,\left(t_{n-1}, z_{n-1}\right)$, $\left(t_{n}, z_{n}\right)=(s, y)$. A comparison of (3.14) and (3.15) yields formula (3.13).

Next consider the general case

$$
X(s, y)=\sum_{n \geq 0} I_{n}\left(f_{n}(\cdot, s, y)\right) .
$$

Define

$$
X_{m}(s, y)=\sum_{n=0}^{m} I_{n}\left(f_{n}(\cdot, s, y)\right), m=1,2, \ldots
$$

Then (3.13) holds for $X_{m}$. Since

$$
\begin{gathered}
\left\|\int_{0}^{\infty} \int_{\mathbb{R}} D_{t, z} X_{m}(s, y) \tilde{N}(\delta s, d y)-\int_{0}^{\infty} \int_{\mathbb{R}} D_{t, z} X(s, y) \tilde{N}(\delta s, d y)\right\|_{L_{2}(P \times \lambda \times \nu)}^{2} \\
=\sum_{n \geq m+1} n^{2} n !\left\|\widehat{f_{n}}\right\|_{L_{2}\left((\lambda \times \nu)^{n+1}\right)}^{2} \longrightarrow 0, \quad m \longrightarrow \infty
\end{gathered}
$$

the proof follows by the closedness of $D_{t, z}$.

The following result is Theorem 4.1 in [NV1] (there obtatined in a more general setting). 
Theorem 3.16 (The Itô-Lévy-Skorohod isometry). Let $X \in L^{2}(P \times \lambda \times \nu)$ and $D X \in$ $L^{2}\left(P \times(\lambda \times \nu)^{2}\right)$. Then the following isometry holds

$$
E\left[\left(\int_{0}^{\infty} \int_{\mathbb{R}} X(t, z) \tilde{N}(\delta t, d z)\right)^{2}\right]
$$

$=E\left[\int_{0}^{\infty} \int_{\mathbb{R}} X^{2}(t, z) \nu(d z) d t\right]+E\left[\int_{0}^{\infty} \int_{\mathbb{R}} \int_{0}^{\infty} \int_{\mathbb{R}} D_{t, z} X(s, y) D_{s, y} X(t, z) \nu(d y) d s \nu(d z) d t\right]$.

Proof. Consider

$$
X(t, z)=\sum_{\alpha \in \mathcal{J}} c_{\alpha}(t, z) K_{\alpha}
$$

Define

$$
S_{1}=\sum_{\alpha \in \mathcal{J}} \alpha !\left\|c_{\alpha}\right\|_{L^{2}(\lambda \times \nu)}^{2}, \quad S_{2}=\sum_{\alpha \in \mathcal{J}, i, j \in \mathbb{N}} \alpha_{\gamma(i, j)} \alpha !\left(c_{\alpha}, \xi_{j} \psi_{i}\right)^{2}
$$

and

$$
S_{3}=\sum_{\substack{\alpha, \beta \in \mathcal{J}, i, j, k, l \in \mathbb{N} \\(i, j) \neq(k, l)}}\left(\alpha_{\gamma(i, j)}+1\right) \alpha !\left(c_{\alpha}, \xi_{j} \psi_{i}\right)\left(c_{\beta}, \xi_{k} \psi_{l}\right) 1_{\left\{\alpha+\epsilon^{\gamma(i, j)}=\epsilon^{\gamma(k, l)}\right\}}
$$

where $(\cdot, \cdot)=(\cdot, \cdot)_{L^{2}(\lambda \times \nu)}$. Note that by the assumption and Lemma 3.12 in $[\varnothing \mathrm{P}]$ the sums above are convergent. First it follows that

$$
\begin{aligned}
E\left[\left(\int_{0}^{\infty} \int_{\mathbb{R}} X(t, z) \widetilde{N}(\delta t, d z)\right)^{2}\right]=E\left[\left(\int_{0}^{\infty} \int_{\mathbb{R}} X(t, z) \diamond \dot{\widetilde{N}}(t, z) \nu(d z) d t\right)^{2}\right] \\
=E\left[\left(\int_{0}^{\infty} \int_{\mathbb{R}}\left(\sum_{\alpha \in \mathcal{J}} c_{\alpha}(t, z) K_{\alpha}\right) \diamond\left(\sum_{i, j} \xi_{j}(t) \psi_{i}(z) K_{\epsilon^{\gamma(i, j)}}\right) \nu(d z) d t\right)^{2}\right] \\
=E\left[\left(\sum_{\alpha \in \mathcal{J}, i, j}\left(c_{\alpha}, \xi_{j} \psi_{i}\right) K_{\alpha+\epsilon^{\gamma(i, j)}}\right)^{2}\right] \\
=\sum_{\substack{\alpha, \beta \in \mathcal{J}, i, j, k, l \in \mathbb{N} \\
(i, j) \neq(k, l)}}\left(\alpha+\epsilon^{\gamma(i, j)}\right) !\left(c_{\alpha}, \xi_{j} \psi_{i}\right)\left(c_{\beta}, \xi_{k} \psi_{l}\right) 1_{\left\{\alpha+\epsilon^{\gamma(i, j)}=\epsilon^{\gamma(k, l)}\right\}}=S_{1}+S_{2}+S_{3},
\end{aligned}
$$

since $\left(\alpha+\epsilon^{\gamma(i, j)}\right) !=\left(\alpha_{\gamma(i, j)}+1\right) \alpha !$.

Next, we have

$$
\begin{gathered}
E\left[\int_{0}^{\infty} \int_{\mathbb{R}} X^{2}(t, z) \nu(d z) d t\right]=E\left[\int_{0}^{\infty} \int_{\mathbb{R}}\left(\sum_{\alpha \in \mathcal{J}} c_{\alpha}(t, z) K_{\alpha}\right)^{2} \nu(d z) d t\right] \\
=\sum_{\alpha \in \mathcal{I}} \int_{0}^{\infty} \int_{\mathbb{R}} c_{\alpha}^{2}(t, z) \alpha ! \nu(d z) d t=S_{1} .
\end{gathered}
$$


Finally, we get

$$
\begin{aligned}
& E\left[\int_{0}^{\infty} \int_{\mathbb{R}} \int_{0}^{\infty} \int_{\mathbb{R}} D_{t, z} X(s, y) D_{s, y} X(t, z) \nu(d y) d s \nu(d z) d t\right] \\
= & E\left[\int_{0}^{\infty} \int_{\mathbb{R}} \int_{0}^{\infty} \int_{\mathbb{R}}\left(\sum_{\alpha, k, l} c_{\alpha}(s, y) \xi_{k}(t) \psi_{l}(z) \alpha_{\epsilon^{\gamma(k, l)}} K_{\alpha-\epsilon^{\gamma(k, l)}}\right)\right. \\
& \left.\cdot\left(\sum_{\beta, i, j} c_{\alpha}(t, z) \xi_{k}(s) \psi_{l}(y) \alpha_{\epsilon^{\gamma(i, j)}} K_{\beta-\epsilon^{\gamma(i, j)}}\right) \nu(d y) d s \nu(d z) d t\right] \\
= & \sum_{\alpha, \beta \in \mathcal{J}, i, j, k, l \in \mathbb{N}}\left(c_{\alpha}, \xi_{j} \psi_{i}\right)\left(c_{\beta}, \xi_{k} \psi_{l}\right) \beta_{\gamma(i, j)} \alpha ! 1_{\left\{\alpha+\epsilon^{\gamma(i, j)}=\epsilon^{\gamma(k, l)}\right\}}=S_{2}+S_{3} .
\end{aligned}
$$

Combining the three steps of the proof the desired result follows.

Remark 3.17 Formula (3.16) can also be obtained as a consequence of the Poisson interpretation of Fock space. See Proposition 17 in [B] and Proposition 1 in [PW]. For an isometry of this type which is not based on Fock space, see Proposition 3.3 in [Pr4].

\section{Forward integrals and generalized Itô formulae.}

In this section we introduce the forward integral with respect to the Poisson random measure $\widetilde{N}$. Then we prove an Itô formula for the corresponding forward processes and we apply this to obtain an Itô formula for processes driven by Skorohod integrals. Here we can refer to $[\mathrm{NP}],[\mathrm{RV}]$ and $[\mathrm{Hu} \varnothing]$, for example, where these topics are developed for the Brownian motion.

Definition 4.1 The forward integral

$$
J(\theta):=\int_{0}^{T} \int_{\mathbb{R}} \theta(t, z) \widetilde{N}\left(d^{-} t, d z\right)
$$

with respect to the Poisson random measure $\widetilde{N}$, of a caglad stochastic function $\theta(t, z), t \in$ $\mathbb{R}_{+}, z \in \mathbb{R}$, with

$$
\theta(t, z):=\theta(\omega, t, z), \quad \omega \in \Omega
$$

is defined as

$$
J(\theta)=\lim _{m \rightarrow \infty} \int_{0}^{T} \int_{\mathbb{R}} \theta(t, z) 1_{U_{m}} \tilde{N}(d t, d z)
$$

if the limit exists in $L^{2}(P)$. Here $U_{m}, m=1,2, \ldots$, is an increasing sequence of compact sets $U_{m} \subseteq \mathbb{R} \backslash\{0\}$ with $\nu\left(U_{m}\right)<\infty$ such that $\lim _{m \rightarrow \infty} U_{m}=\mathbb{R} \backslash\{0\}$.

Remark 4.2 Note that if $G$ is a random variable then

$$
G \cdot \int_{0}^{T} \int_{\mathbb{R}} \theta(t, z) \tilde{N}\left(d^{-} t, d z\right)=\int_{0}^{T} \int_{\mathbb{R}} G \cdot \theta(t, z) \tilde{N}\left(d^{-} t, d z\right),
$$

a property that does not hold for the Skorohod integrals. 
Definition 4.3 In the sequel we let $\mathcal{M}$ denote the set of the stochastic functions $\theta(t, z)$, $t \in \mathbb{R}_{+}, z \in \mathbb{R}$, such that

(i) $\theta(\omega, t, z)=\theta_{1}(\omega, t) \theta_{2}(\omega, t, z)$ where $\theta_{1}(\omega, t) \in \mathbb{D}_{1,2}$ is caglag and $\theta_{2}(\omega, t, z)$ is adapted and such that

$$
E\left[\int_{0}^{T} \int_{\mathbb{R}} \theta_{2}^{2}(t, z) \nu(d z) d t\right]<\infty
$$

(ii) $D_{t^{+}, z} \xi=\lim _{s \rightarrow t^{+}} D_{s, z} \xi$ exists in $L^{2}(P \times \lambda \times \nu)$,

(iii) $\theta(t, z)+D_{t^{+}, z} \theta(t, z)$ is Skorohod integrable.

We let $\mathbb{M}_{1,2}$ be the closure of the linear span of $\mathcal{M}$ with respect to the norm given by

$$
\|\theta\|_{\mathbb{M}_{1,2}}^{2}:=\|\theta\|_{L^{2}(P \times \lambda \times \nu)}^{2}+\left\|D_{t^{+}, z} \theta(t, z)\right\|_{L^{2}(P \times \lambda \times \nu)}^{2} .
$$

We can now show the relation between the forward integral and the Skorohod integral.

Lemma 4.4 If $\theta \in \mathbb{M}_{1,2}$ then its forward integral exists and

$\int_{0}^{T} \int_{\mathbb{R}} \theta(t, z) \tilde{N}\left(d^{-} t, d z\right)=\int_{0}^{T} \int_{\mathbb{R}} D_{t^{+}, z} \theta(t, z) \nu(d z) d t+\int_{0}^{T} \int_{\mathbb{R}}\left(\theta(t, z)+D_{t^{+}, z} \theta(t, z)\right) \tilde{N}(\delta t, d z)$.

Proof. First consider the case when $\theta(\omega, t, z)=\theta_{1}(\omega, t) \theta_{2}(\omega, t, z)$. Let us take a sequence of partitions of $[0, T]$ of the form $0=t_{0}^{n}<t_{1}^{n}<\ldots<t_{J_{n}}^{n}=T$ with $|\Delta t|:=\max \left(t_{j}^{n}-t_{j-1}^{n}\right) \longrightarrow 0$, for $n \rightarrow \infty$, into account. By Theorem 3.13 we have

$$
\begin{gathered}
F \cdot \int_{t_{i-1}^{n}}^{t_{i}^{n}} \int_{\mathbb{R}} \theta(t, z) \widetilde{N}(\delta t, d z)=\int_{t_{i-1}^{n}}^{t_{i}^{n}} \int_{\mathbb{R}} F \theta(t, z) \widetilde{N}(\delta t, d z) \\
+\int_{t_{i-1}^{n}}^{t_{i}^{n}} \int_{\mathbb{R}} \theta(t, z) D_{t, z} F \nu(d z) d t+\int_{t_{i-1}^{n}}^{t_{i}^{n}} \int_{\mathbb{R}} \theta(t, z) D_{t, z} F \widetilde{N}(\delta t, d z) .
\end{gathered}
$$

Hence

$$
\begin{gathered}
\int_{0}^{T} \int_{\mathbb{R}} \theta(t, z) \tilde{N}\left(d^{-} t, d z\right)=\lim _{|\Delta t| \longrightarrow 0} \sum_{i=1}^{J_{n}} \theta_{1}\left(t_{i-1}^{n}\right) \int_{t_{i-1}^{n}}^{t_{i}^{n}} \int_{\mathbb{R}} \theta_{2}(t, z) \tilde{N}(d t, d z) \\
=\lim _{|\Delta t| \longrightarrow 0} \sum_{i=1}^{J_{n}} \theta_{1}\left(t_{i-1}^{n}\right) \int_{t_{i-1}^{n}}^{t_{i}^{n}} \int_{\mathbb{R}} \theta_{2}(t, z) \tilde{N}(\delta t, d z) \\
=\lim _{|\Delta t| \longrightarrow 0} \sum_{i=1}^{J_{n}} \int_{t_{i-1}^{n}}^{t_{i}^{n}} \int_{\mathbb{R}}\left[\theta_{1}\left(t_{i-1}^{n}\right)+D_{t, z} \theta_{1}\left(t_{i-1}^{n}\right)\right] \theta_{2}(t, z) \widetilde{N}(\delta t, d z) \\
\quad+\lim _{|\Delta t| \longrightarrow 0} \sum_{i=1}^{J_{n}} \int_{t_{i-1}^{n}}^{t_{i}^{n}} \int_{\mathbb{R}} D_{t, z} \theta_{1}\left(t_{i-1}^{n}\right) \cdot \theta_{2}(t, z) \nu(d z) d t \\
=\int_{0}^{T} \int_{\mathbb{R}} \theta(t, z) \tilde{N}(\delta t, d z)+\int_{0}^{T} \int_{\mathbb{R}} D_{t^{+}, z} \theta(t, z) \nu(d z) d t+\int_{0}^{T} \int_{\mathbb{R}} D_{t^{+}, z} \theta(t, z) \tilde{N}(\delta t, d z) .
\end{gathered}
$$

The proof is then completed by a limit argument in view of Definition 3.10 and Definition 4.3 . 
Corollary 4.5 If the forward integral exists in $L^{2}(P)$ then

$$
E \int_{0}^{T} \int_{\mathbb{R}} \theta(t, z) \tilde{N}\left(d^{-} t, d z\right)=E \int_{0}^{T} \int_{\mathbb{R}} D_{t^{+}, z} \theta(t, z) \nu(d z) d t .
$$

Proof. This follows from (3.3) and Lemma 4.4.

Definition 4.6 A forward process is a measurable stochastic function $X(t)=X(t, \omega), t \in$ $\mathbb{R}_{+}, \omega \in \Omega$, that admits the representation

$$
X(t)=x+\int_{0}^{t} \int_{\mathbb{R}} \theta(s, z) \widetilde{N}\left(d^{-} s, d z\right)+\int_{0}^{t} \alpha(s) d s
$$

where $x=X(0)$ is a constant. A shorthand notation for (4.3) is

$$
d^{-} X(t)=\int_{\mathbb{R}} \theta(t, z) \widetilde{N}\left(d^{-} t, d z\right)+\alpha(t) d t ; \quad X(0)=x .
$$

We call $d^{-} X(t)$ the forward differential of $X(t), t \in \mathbb{R}_{+}$.

Theorem 4.7 (Itô formula for forward integrals). Let $X(t), t \in \mathbb{R}_{+}$, be a forward process of the form (4.3) where $\theta(t, z), t \in \mathbb{R}_{+}, z \in \mathbb{R}$, is locally bounded in $z$ near $z=0$ $P \times \lambda$-a.e. and such that

$$
\int_{0}^{T} \int_{\mathbb{R}}|\theta(t, z)|^{2} \nu(d z) d t<\infty \quad P-a . s .
$$

Suppose also that $|\theta(t, z)|, t \in \mathbb{R}_{+}, z \in \mathbb{R}$, is forward integrable. For any function $f \in C^{2}(\mathbb{R})$, the forward differential of $Y(t)=f(X(t)), t \in \mathbb{R}_{+}$, is given by the following formula:

$$
\begin{gathered}
d^{-} Y(t)=f^{\prime}(X(t)) \alpha(t) d t \\
+\int_{\mathbb{R}}\left(f\left(X\left(t^{-}\right)+\theta(t, z)\right)-f\left(X\left(t^{-}\right)\right)-f^{\prime}\left(X\left(t^{-}\right)\right) \theta(t, z)\right) \nu(d z) d t \\
+\int_{\mathbb{R}}\left(f\left(X\left(t^{-}\right)+\theta(t, z)\right)-f\left(X\left(t^{-}\right)\right)\right) \tilde{N}\left(d^{-} t, d z\right) .
\end{gathered}
$$

Proof. The proof follows the same line of the one in the classical Itô formula (see [IW] Chapter 2, Section 5). For simplicity we assume $x=0$ and $\alpha \equiv 0$. We can write

$$
X_{m}(t):=\int_{0}^{t} \int_{\mathbb{R}} \theta(s, z) 1_{U_{m}}(z) N(d s, d z)-\int_{0}^{t} \int_{\mathbb{R}} \theta(s, z) 1_{U_{m}}(z) \nu(d z) d s .
$$

We denote by $0=\sigma_{0}<\sigma_{1}<\ldots$ the stopping times for which the jumps of the Lévy process occur. Thus we obtain

$$
f\left(X_{m}(t)\right)-f\left(X_{m}(0)\right)=\sum_{i}\left[f\left(X_{m}\left(\sigma_{i} \wedge t\right)\right)-f\left(X_{m}\left(\sigma_{i} \wedge t^{-}\right)\right)\right]
$$




$$
+\sum_{i}\left[f\left(X_{m}\left(\sigma_{i} \wedge t^{-}\right)\right)-f\left(X_{m}\left(\sigma_{i-1} \wedge t\right)\right)\right]=: \mathcal{J}_{1}(t)+\mathcal{J}_{2}(t)
$$

with

$$
f\left(X_{m}\left(\sigma_{i} \wedge t^{-}\right)\right)=\left\{\begin{array}{l}
f\left(X_{m}\left(\sigma_{i}^{-}\right)\right), \quad \sigma_{i} \leq t \\
f\left(X_{m}(t)\right), \quad \sigma_{i}>t
\end{array}\right.
$$

By the change of variable formula for finite variation processes, it follows that

$$
\mathcal{J}_{2}(t)=-\int_{0}^{t} \int_{\mathbb{R}} f^{\prime}\left(X_{m}(s)\right) \theta(s, z) 1_{U_{m}}(z) \nu(d z) d s .
$$

Moreover it is

$$
\begin{aligned}
& \mathcal{J}_{1}(t)=\sum_{i}\left[f\left(X_{m}\right)\left(\sigma_{i}\right)-f\left(X_{m}\right)\left(\sigma_{i}^{-}\right)\right] 1_{\left\{\sigma_{i} \leq t, \theta\left(\sigma_{i}, \eta\left(\sigma_{i}\right)\right) \neq 0\right\}} \\
= & \int_{0}^{t} \int_{\mathbb{R}}\left[f\left(X_{m}\left(s^{-}\right)+\theta(s, z) 1_{U_{m}}(z)\right)-f\left(X_{m}\right)\left(s^{-}\right)\right] N(d s, d z) \\
= & \int_{0}^{t} \int_{\mathbb{R}}\left[f\left(X_{m}\left(s^{-}\right)+\theta(s, z) 1_{U_{m}}(z)\right)-f\left(X_{m}\right)\left(s^{-}\right)\right] \widetilde{N}\left(d^{-} s, d z\right) \\
+ & \int_{0}^{t} \int_{\mathbb{R}}\left[f\left(X_{m, n}\left(s^{-}\right)+\theta(s, z) 1_{U_{m}}(z)\right)-f\left(X_{m}\right)\left(s^{-}\right)\right] \nu(d z) d s .
\end{aligned}
$$

By letting $m \rightarrow \infty$, formula (4.6) follows.

In order to state an Itô formula for Skorohod integrals we need to combine Lemma 4.4 and Theorem 4.7. To this end we go into the technical step of solving equations of the following type: given a random variable $G$ find the stochastic function $F(t, z), t \in \mathbb{R}_{+}, z \in \mathbb{R}$, such that

$$
F(t, z)+D_{t^{+}, z} F(t, z)=G,
$$

for almost all $(t, z) \in \mathbb{R}_{+} \times \mathbb{R}$. For example, if $G=g(\eta(T))$, for some measurable function $g: \mathbb{R} \longrightarrow \mathbb{R}$ and

$$
\eta(t)=\int_{0}^{t} \int_{\mathbb{R}} z \tilde{N}(d t, d z), \quad t \in[0, T]
$$

then

$$
F(t, z):=g\left(\eta(T)-z \chi_{[0, T)}(t)\right)
$$

does the job. In fact, with this choice of $F(t, z), t \in \mathbb{R}_{+}, z \in \mathbb{R}$, we have

$$
F(t, z)+D_{t^{+}, z} F(t, z)=g\left(\eta(T)-z \chi_{[0, T)}\right)+g(\eta(T))-g\left(\eta(T)-z \chi_{[0, T)}\right)=G .
$$

The above observation motivates the following definition.

Definition 4.8 The linear operator $S$ is defined on the space of all $\mathcal{F}_{T}$-measurable random variables $G$ as follows. If $G=\prod_{i=1}^{k} g_{i}\left(\eta\left(t_{i}\right)\right)$, for some $t_{i} \in[0, T], i=1, \ldots, k$, we define

$$
S_{t, z}\left(\prod_{i=1}^{k} g_{i}\left(\eta\left(t_{i}\right)\right)\right)=\prod_{i=1}^{k} g_{i}\left(\eta\left(t_{i}\right)-z \chi_{\left[0, t_{i}\right)}(t)\right) .
$$


Note that via this definition the solution of equation (4.6) can be written as $F(t, z)=$ $S_{t, z} G$, i.e.

$$
S_{t, z} G+D_{t^{+}, z}\left(S_{t, z} G\right)=G \text {. }
$$

Combining the above facts with Lemma 4.4 and Theorem 4.7, we obtain the following result.

Theorem 4.9 (Itô formula for Skorohod integrals). Let

$$
X(t)=\int_{0}^{t} \int_{\mathbb{R}} \gamma(s, z) \widetilde{N}(\delta s, d z)+\int_{0}^{t} \alpha(s) d s, \quad t \in[0, T]
$$

or, in shorthand notation,

$$
\delta X(t)=\int_{\mathbb{R}} \gamma(t, z) \widetilde{N}(\delta t, d z)+\alpha(t) d t, \quad t \in[0, T] .
$$

Let $f \in C^{2}(\mathbb{R})$ and let $Y(t)=f(X(t))$. Set

$$
\theta(t, z):=S_{t, z} \gamma(t, z)
$$

for all $t \in[0, T], z \in \mathbb{R}$, and assume $\theta \in \mathbb{M}_{1,2}$. Then

$$
\begin{gathered}
\delta Y(t)=f^{\prime}(X(t)) \alpha(t) d t+\int_{\mathbb{R}}\left\{f\left(X\left(t^{-}\right)+\theta(t, z)\right)-f\left(X\left(t^{-}\right)\right)\right. \\
\left.+D_{t^{+}, z}\left[f\left(X\left(t^{-}\right)+\theta(t, z)\right)-f\left(X\left(t^{-}\right)\right)\right]\right\} \widetilde{N}(\delta t, d z) \\
+\int_{\mathbb{R}}\left\{f\left(X\left(t^{-}\right)+\theta(t, z)\right)-f\left(X\left(t^{-}\right)\right)-f^{\prime}\left(X\left(t^{-}\right)\right) \theta(t, z)\right. \\
\left.+D_{t^{+}, z}\left[f\left(X\left(t^{-}\right)+\theta(t, z)\right)-f\left(X\left(t^{-}\right)\right)\right]-f^{\prime}\left(X\left(t^{-}\right)\right) D_{t^{+}, z} \theta(t, z)\right\} \nu(d z) d t .
\end{gathered}
$$

Remark 4.10 Note that if $\gamma$ and $\alpha$ are adapted, then $\theta(t, z)=\gamma(t, z), t \in \mathbb{R}_{+}, z \in \mathbb{R}$, and

$$
D_{t^{+}, z} \theta(t, z)=D_{t^{+}, z}\left[f\left(X\left(t^{-}\right)+\theta(t, z)\right)-f\left(X\left(t^{-}\right)\right)\right] .
$$

Therefore Theorem 4.9 reduces to the classical adapted Itô formula.

Proof. For simplicity we assume $\alpha \equiv 0$. By (4.8) we have

$$
\theta(t, z)+D_{t^{+}, z} \theta(t, z)=\gamma(t, z) .
$$

Hence by Lemma 4.4 we have

$$
X(t)=\int_{0}^{t} \int_{\mathbb{R}} \theta(s, z) \tilde{N}\left(d^{-} s, d z\right)-\int_{0}^{t} \int_{\mathbb{R}} D_{s^{+}, z} \theta(s, z) \nu(d z) d s .
$$


We can therefore apply Theorem 4.7 and get

$$
\begin{gathered}
Y(t)-Y(0)=\int_{0}^{t} f^{\prime}(X(s))\left(-\int_{\mathbb{R}} D_{s^{+}, z} \theta(s, z) \nu(d z)\right) d s \\
+\int_{0}^{t} \int_{\mathbb{R}}\left\{f\left(X\left(s^{-}\right)+\theta(s, z)\right)-f\left(X\left(s^{-}\right)\right)-f^{\prime}\left(X\left(s^{-}\right)\right) \theta(s, z)\right\} \nu(d z) d s \\
+\int_{0}^{t} \int_{\mathbb{R}}\left\{f\left(X\left(s^{-}\right)+\theta(s, z)\right)-f\left(X\left(s^{-}\right)\right)\right\} \tilde{N}\left(d^{-} s, d z\right) \\
-\int_{0}^{t} \int_{\mathbb{R}} f^{\prime}\left(X\left(s^{-}\right)\right) D_{s^{+}, z} \theta(s, z) \nu(d z) d s \\
+\int_{0}^{t} \int_{\mathbb{R}}\left\{f\left(X\left(s^{-}\right)+\theta(s, z)\right)-f\left(X\left(s^{-}\right)\right)-f^{\prime}\left(X\left(s^{-}\right)\right) \theta(s, z)\right\} \nu(d z) d s \\
+\int_{0}^{t} \int_{\mathbb{R}} D_{s^{+}, z}\left\{f\left(X\left(s^{-}\right)+\theta(s, z)\right)-f\left(X\left(s^{-}\right)\right)\right\} \nu(d z) d t \\
+\int_{0}^{t} \int_{\mathbb{R}}\left\{f\left(X\left(s^{-}\right)+\theta(s, z)\right)-f\left(X\left(s^{-}\right)\right)+D_{s^{+}, z}\left\{f\left(X\left(s^{-}\right)+\theta(s, z)\right)-f\left(X\left(s^{-}\right)\right)\right\}\right\} \tilde{N}(\delta s, d z) \\
=\int_{0}^{t} \int_{\mathbb{R}}\left\{f\left(X\left(s^{-}\right)+\theta(s, z)\right)-f\left(X\left(s^{-}\right)\right)-f^{\prime}\left(X\left(s^{-}\right)\right) \theta(s, z)\right. \\
\left.+D_{s^{+}, z}\left[f\left(X\left(s^{-}\right)+\theta(s, z)\right)-f\left(X\left(s^{-}\right)\right)\right]-f f^{\prime}\left(X\left(s^{-}\right)\right) D_{s^{+}, z} \theta(s, z)\right\} \nu(d z) d s \\
+\int_{0}^{t} \int_{\mathbb{R}}\left\{f\left(X\left(s^{-}\right)+\theta(s, z)\right)-f\left(X\left(s^{-}\right)\right)+D_{s^{+}, z}\left[f\left(X\left(s^{-}\right)+\theta(s, z)\right)-f\left(X\left(s^{-}\right)\right)\right]\right\} \widetilde{N}(\delta s, d z) .
\end{gathered}
$$

This completes the proof.

Remark 4.11 In [Pr1] a different anticipative Itô formula is obtained, valid for polynomials $f$.

Remark 4.12 The Itô formula can be extended to cover the mixed case, involving a combination of Gaussian and compensated Poisson random measures.

\section{References}

[A] Applebaum, D.: Covariant Poisson fields in Fock space. In Analysis, Geometry and Probability. Hindustan Book Agency, Delhi 1996, pp. 1-15.

[AØPU] Aase, K., Øksendal, B., Privault, N., Ubøe, J.: White noise generalizations of the ClarkHaussmann-Ocone theorem with application to mathematical finance. Finance and Stochastics 4 (2000), pp. 465-496.

[B] Biane, P.:Calcul Stochastic non-commutatif. Lectures on Probability Theory (Saint-Flour, 1993). Lect. Notes in Math. 1608. Springer 1995, pp. 1-96. 
[BC] Bass, R.F., Cranston, M.: The Malliavin calculus for pure jump processes and applications to local time. Annals. Prob. 14 (1986), pp. 490-532.

[BG] Benth, F.E., Gjerde, J.: A remark on the equivalence between Poisson and Gaussian stochastic partial differential equations. Pot. Anal. 8 (1998), pp. 179-193.

[BL] Benth, F.E., Løkka, A.: Anticipative calculus for Lévy processes and stochastic differential equations. Preprint Series in Pure Mathematics, University of Oslo, 6, 2002.

[BDLØP] Benth, F.E., di Nunno, G., Løkka, A., Øksendal, B., Proske, F.: Explicit representation of the minimal variance portfolio in markets driven by Lévy processes. Math. Finance 13 (2003), pp.54-72.

[Be] Bertoin, J.: Lévy Processes. Cambridge University Press, Cambridge 1996.

[BØ1] Biagini, F., Øksendal, B.: A general stochastic caluculus approach to insider trading. Preprint Series in Pure Mathematics, University of Oslo, 17, 2002.

[BØ2] Biagini, F., Øksendal, B.: Minimal variance hedging for insider trading. Preprint Series in Pure Mathematics, University of Oslo, 9, 2004.

[BGJ] Bichteler, K., Gravereaux, J.B., Jacod, J.: Malliavin Calculus for Processes with Jumps. Gordon and Breach Science Publisher, New York, 1987.

[DJ] Davis, M.H.A., Johansson, M.P.: Malliavin Monte Carlo greeks for jump diffusions. Preprint Imperial College, June 2004.

[DKW] Dermoune, A., Kree, P., Wu, L.: Calcul stochastique non adapté par rapport à la mesure aléatoire de Poisson. Séminaire de Probabilités XXII, Lect. Notes. Math 1321, pp. 477-484, Springer, Berlin, 1988.

[DMØP] di Nunno, G., Meyer-Brandis, T., Øksendal, B., Proske, F.: Optimal portfolio for an insider in a market driven by Lévy processes. Preprint Series in Pure Mathematics, University of Oslo, 36, 2003.

[DØP] di Nunno, G., Øksendal, B., Proske, F.: White noise analysis for Lévy proceses. Journal of Functional Analysis 206 (2004), pp. 109-148.

[FLLLT] Fournié, E., Larry, J.M., Lebuchoux, J., Lions, P.L., Touzi, N.: Applications of Malliavin calculus to Monte Carlo methods in finance. Finance Stoch. 3 (1999), pp. 391-412.

[FLLL] Fournié, E., Larry, J.M., Lebuchoux, J., Lions, P.L.: Applications of Malliavin calculus to Monte Carlo methods in finance II. Finance Stoch. 5 (2001), pp. 201-236.

[GV] Gelfand, I. M., Vilenkin, N. Y.: Generalized Functions, Vol. 4: Applications of Harmonic Analysis. Academic Press (English translation), New York-London 1964.

[HKPS] Hida, T., Kuo, H.-H, Potthoff, J., Streit, L.: White Noise. Kluwer, Dordrecht, 1993.

[HuØ] Hu, Y., Øksendal, B.: Optimal smooth portfolio selection for an insider. Preprint Series in Pure Mathematics, University of Oslo, 12, 2003.

[HoØ] Holden, H., Øksendal, B.: A white noise approach to stochastic differential equations driven by Wiener and Poisson processes. In M. Grosser et al. (editors): Nonlinear Theory of Generalized Functions, Chapman \& Hall/CRC 1999, pp 293-313. 
[HoØUZ] Holden, H., Øksendal, B., Ubøe, J., Zhang T.-S.: Stochastic Partial Differential Equations:A Modeling, White Noise Functional Approach. Birkhäuser, Boston 1996.

[IW] Ikeda, N., Watanabe, S.: Stochastic Differential Equations and Diffusion Processes. NorthHolland/Kondansha LTD., Tokyo 1989.

[I1] Itô, K.: On stochastic processes I. Infinitely divisible laws of probability. Jap. J. Math. 18 (1942), pp. $252-301$.

[I2] Itô, K.: Spectral type of the shift transformation of differential processes with stationary increments, Trans. Am. Math. Soc. 81 (1956), pp. 253-263.

[Ka1] Kabanov, Y.: A generalized Itô formula for an extended stochastic integral with respect to a Poisson random measure (In Russian), Usp. Mat. Nauk. 29 (1974), No. 4 (178), pp. 167-168.

[Ka2] Kabanov, Y.: On extended stochastic integrals. Theory of Probability and its Applications 20 (1975), pp. $710-722$.

[K1] Kaminsky, A.D.: Extended stochastic clalculus for the Poisson random measures. Nats. Akad. Nauk Ukrain, Inst. Mat. Preprint, 15 (1996).

[K2] Kaminsky, A.D.: A white noise approach to stochastic integration for a Poisson random measure. Theory Probab. Math. Statist. 57 (1998), pp. 43-52.

[KO] Karatzas, I., Ocone, D.: A generalized Clark representation formula, with application to optimal portfolios. Stochastics and Stochastic Reports 34 (1991), pp. 187-220.

[KS] Kohatsu-Higa, A., Sulem, A.: Utility maximization in an insider influenced market. Preprint INRIA, September 2004.

[LSUV] Léon, J.A., Solé, J.L., Utzet, F., Vives, J.: On Lévy processes, Malliavin calculus and market models with jumps. Prepublications 2001, U.A.B., Barcelona. To appear in Finance and Stochastics.

[L] Løkka, A.: Martingale representation and functionals of Lévy processes. Preprint Series in Pure Mathematics, University of Oslo, 21, 2001.

[M] Malliavin, P.: Stochastic Analysis. Springer-Verlag, New York 1997.

[N] Nualart, D.: The Malliavin Calculus and Related Topics. Springer, Berlin Heidelberg New York 1995.

[NP] Nualart, D., Pardoux E.: Stochastic calculus with anticipating integrands. Probab. Th. Rel. Fields 78 (1988), pp. 535-581.

[NS] Nualart, D., Schoutens, W.: Chaotic and predictable representations for Lévy processes. Stochastic Process. Appl. 90 (2000), pp. 109-122.

[NV1] Nualart, D., Vives, J.: Anticipative calculus for the Poisson process based on the Fock space, Séminaire de Probabilités XXIV, LNM 1426, 1990.

[NV2] Nualart, D., Vives, J.: A duality formula on the Poisson space and some applications. Seminar on Stochastic Analysis, Random Fields and Applications (Ascona, 1993), pp. 205-213, Progr. Probab., 36, Birkhuser, Basel, 1995. 
[Ø] Øksendal, B.: An introduction to Malliavin calculus with applications to economics. Working paper, No 3/96, Norwegian School of Economics and Business Administration, 1996.

[Ø2] Øksendal, B.: A universal optimal consumption rate for an insider. Preprint Series in Pure Mathematics, University of Oslo, 27, 2004.

[ØР] Øksendal, B., Proske, F.: White noise for Poisson random measures. Preprint Series in Pure Mathematics, University of Oslo, 12, 2002.

[ØS] Øksendal, B., Sulem, A.: Partial observation control in an anticipating environment. Preprint Series in Pure Mathematics, University of Oslo, 31, 2003.

[Pi1] Picard, J.: On the existence of smooth densities for jump processes. Prob. Th. Rel. Fields 105 (1996), pp. 481-511.

[Pi2] Picard, J.: Formules de dualité sur l'espace de Poisson. Ann. IHP, Série Proba. et Stat. 32 (1996), pp. 509-548.

[Pa] Parthasarathy, K.R.: An Introduction to Quantum Stochastic Calculus. Birkäuser 1992.

[Pr1] Privault, N.: An extension of stochastic calculus to certain non-Markovian processes. Manuscript 1997.

[Pr2] Privault, N.: Independence of a class of multiple stochastic integrals. In Seminar on Stochastic Analysis, Random Fields and Applications (Ascona 1996). Birkäuser 1999, pp. 249-259.

[Pr3] Privault, N.: Equivalence of gradients on configuration spaces. Random Oper. Stoch. Equations 7 (1999), pp. 241-262.

[Pr4] Privault, N.: Connections and curvature in the Riemannian geometry of configuration spaces. Journal of Functional Analysis 185 (2001), pp. 367-403.

[PSV] Privault, N., Solé, J.L. and Vives, J.: Chaotic Kabanov formula for the Azma martingales. Bernoulli 6 (2000), pp. 633-651.

[PW] Privault, N. and Wu, J.: Poisson stochastic integration in Hilbert spaces. Ann. Math. Blaise Pascal 6 (1999), pp. 41-61.

[PT] Potthoff, J., Timpel, M.: On a dual pair of smooth and generalized random variables. Potential Analysis 4 (1995), pp. 637-654.

[P] Protter, P.: Stochastic Integration and Differential Equations. Springer-Verlag, Berlin 1990.

[RV] Russo, F., Vallois, P.: Forward, backward and symmetric stochastic integration. Prob. Th. Rel. Fields 97 (1993), pp. 403-421.

[Sa] Sato, K.: Lévy Processes and Infinitely Divisible Distributions, Cambridge University Studies in Advanced Mathematics, Vol. 68, Cambridge University Press, Cambridge 1999.

[Su] Surgailis, D.:On multiple Poisson stochastic integrals and associated Markov semigroups. Probab. Math. Statist. 3 (1984), pp. 217-239.

[T] Thangavelu, S.: Lectures on Hermite and Laguerre Expansions. Mathematical Notes 42, Princeton University Press 1993. 